\section{Liver failure}

\section{PTU-001 PROPANOLOL AT MODEST DOSE DOES NOT IMPAIR SURVIVAL IN PATIENTS WITH CIRRHOSIS AND REFRACTORY ASCITES}

doi:10.1136/gutjnl-2012-302514c.1

A E Robins, ${ }^{*}$ A Bowden, W Watson, F Smith, W T Gelson, W J Griffiths. Department of Hepatology, Cambridge University Hospitals, Cambridge, UK

Introduction A recent study suggesting $\beta$-blockers should be contraindicated in cirrhotic patients with refractory ascites has gained significant attention. ${ }^{1}$ We sought to examine this hypothesis in a cohort of patients undergoing elective paracentesis in a tertiary liver unit.

Methods We retrospectively studied 114 consecutive patients undergoing regular paracentesis between July 2007 and December 2010 at Addenbrooke's Hospital. 36 patients were maintained on propanolol for variceal prophylaxis, whereas 78 were not $\beta$-blocked. Mortality and morbidity were compared between the two groups. The $\chi^{2}$, Mann-Whitney and Kaplan-Meier methods were employed for statistical analysis.

Results There was no statistically significant difference between the two groups in terms of age, sex, aetiology of liver disease (predominantly alcohol), Child-Pugh score and UKELD. Hepatocellular carcinoma was present in $16 \%$ of patients in the propanolol group and $13 \%$ of the non $\beta$-blocked group $(p=0.62)$. Varices were predictably present more in the propanolol group compared with the non $\beta$-blocked group ( $97 \%$ vs $59 \%, \mathrm{p}<0.001)$. The mean total daily dose of propanolol used was $48.9 \mathrm{mg}$. The incidence of spontaneous bacterial peritonitis was similar between the propanolol group and non $\beta$-blocked group ( $43 \%$ vs $50 \%, \mathrm{p}=0.51$ ). The incidence of overt encephalopathy was also no different (43\% vs $44 \%$, $\mathrm{p}=0.93$ ). Variceal bleeding occurred more frequently in the propanolol group compared with the non $\beta$-blocked group (69\% vs $41 \%$, $\mathrm{p}<0.01)$. Median survival was 18 months in the propranolol group vs 11 months in the non-propanolol group, with no significant difference between Kaplan-Meier survival curves ( $p=0.93$, log rank test) (Abstract PTU-001 figure 1).

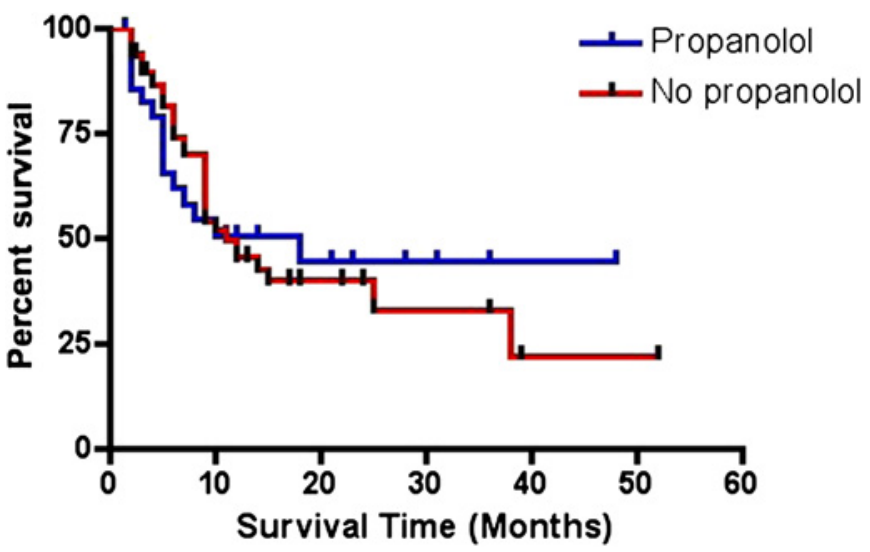

Abstract PTU-001 Figure 1 Kaplan-Meier survival curve.

Conclusion This study demonstrates that propanolol used in a total daily dose of between 40 and $80 \mathrm{mg}$ is safe in patients with cirrhosis and refractory ascites. Deleterious effects at higher doses cannot be excluded. Notwithstanding this limitation and the retrospective nature of the analysis, these data reassure regarding the use of $\beta$ blockers in this patient group and that such drugs should not be immediately contraindicated.
Competing interests None declared.

\section{REFERENCE}

1. Serste T, Melot C, Francoz C, et al. Deleterious effects of beta-blockers on survival in patients with cirrhosis and refractory ascites. Hepatology 2010;52:1017-22.

\section{PTU-002 THE HEPATIC INFLAMMATORY MILIEU OF ACETAMINOPHEN INDUCED ACUTE LIVER FALURE INDUCES ALTERNATIVELY ACTIVATED, M2-LIKE, MACROPHAGES}

doi:10.1136/gutjnl-2012-302514c.2

${ }^{1,2} \mathrm{C}$ G Antoniades, ${ }^{*}{ }^{2} \mathrm{~V}$ Zingarelli, ${ }^{3} \mathrm{~L}$ Possamai, ${ }^{1} \mathrm{~W}$ Khamri, ${ }^{1} \mathrm{~N}$ Vergis, ${ }^{2} \mathrm{R}$ Mitry, ${ }^{2} \mathrm{~A}$ Ouaglia, ${ }^{2} \mathrm{~W}$ Bernal, ${ }^{2} \mathrm{G}$ Auzinger, ${ }^{2} \mathrm{Y} \mathrm{Ma},{ }^{2} \mathrm{~J}$ Wendon, ${ }^{1} \mathrm{M}$ Thursz. ${ }^{1}$ Section of Hepatology, Imperial College London, London, UK; ${ }^{2}$ Institute of Liver Studies, King's College London, London, UK; ${ }^{3}$ Section of Hepatology, Imperial College Hospital, London, UK

Introduction Human acetaminophen-induced acute liver injury (AALF) is characterised by areas of hepatic necrosis that are infiltrated by macrophages $(\mathrm{m} \varphi)$ and elevated concentrations of antiinflammatory cytokines. Anti-inflammatory mediators, such as secretory leucoprotease inhibitor (SLPI) and IL-10 contribute to the functional switching of $\mathrm{m} \varphi$ from a proinflammatory (M1) type to an alternatively activated (M2) phenotype that promotes tissue repair processes. We sought to determine the effects of the hepatic inflammatory milieu on peripheral monocytes (MO) and $\mathrm{MO}$ derived $m \varphi$ in AALF.

Methods Phosphoflow technique was used to evaluate NF-kBp65, STAT-3 signalling pathways in ex-vivo $\mathrm{MO}$ in 10 AALF patients and 10 healthy controls (HC). Results expressed as MFI and ratio of activation. Regional changes (portal vein [PV]), hepatic vein [HV])) were assessed in 5 AALF patients at time of transplantation. Serum (AALF [ $n=34]$; HC $[n=15]$ ), hepatic (AALF [ $n=7]$; HC $[n=8]$ ) and regional levels of TNF- $\alpha$, IL-10 and SLPI were measured. The effect of the hepatic microenvironment was assessed in five cell culture experiments: purified $\mathrm{CD} 14+\mathrm{MO}$ from $\mathrm{HC}$ were incubated with homogenates from AALF and culture medium (CM).

Results In MO, TLR-4 stimulation reduced NF-kBp65 expression in AALF compared to HC (0.8 vs 1.6; $p=0.001)$. Ex-vivo STAT-3 expression was significantly elevated in AALF patients compared to HC (600 vs 232; $p=0.02)$. AALF patients had higher serum concentrations of IL-10 (170 vs 40; $p<0.02$ ), SLPI (71200 vs 43310 ; $\mathrm{p}<0.0001)$ compared to HC. A trans-hepatic $(\mathrm{HV}>\mathrm{PV})$ gradient was seen for IL-10 and SLPI but not for TNF- $\alpha$. Hepatic levels of IL-10 (2 vs $0.6 ; \mathrm{p}<0.02)$ and SLPI (442 vs $116 ; \mathrm{p}<0.01$ ) were significantly elevated in AALF compared to HC tissue, with peak concentrations of IL-10 detected in necrotic areas while SPLI was highest in areas of hepatic regeneration. In vitro exposure to AALF milieu induced a unique $\mathrm{CD} 14+\mathrm{CD} 16+$ macrophage phenotype characterised increased expression of alternative (M2) activation markers- CD36 (78 vs $55 \% ; p=0.01$ ) and CD163 (58 vs $32.5 \% ; p=0.04$ ), reduced LPSinduced TNF-a (19.8 vs 40.5\%; $p=0.02)$, IL-6 (13.2 vs $22 \%$; $p=0.04)$ and enhanced phagocytosis ( 80.5 vs $70 \%$; $=0.03$ ) when compared to $\mathrm{MO}$ incubated $\mathrm{CM}$.

Conclusion In AALF, circulating monocytes show modulations in intracellular signalling pathways compatible with anti-inflammatory responses. Our data also indicate that the anti-inflammatory hepatic microenvironment preferentially induces alternatively activated (M2)-like macrophages that may be implicated in resolution of acute liver injury.

Competing interests None declared. 